\section{Techniques to preserve keratinized peri-implant mucosa in CT-guided oral implant surgery}

\section{Bernhard Pommer}

Department of Oral Surgery, Vienna Medical University, Austria

\section{Abstract}

Guided implant dentistry using computed tomographic (CT) scans, virtual planning software and mucosa-supported surgical templates is an upcoming technology with the potential for more predictable and less invasive implant placement. While generally associated with a flapless approach, soft tissue punching and removal may not be indicated if available width of keratinized mucosa is limited prior to implant surgery. Two techniques to preserve keratinized peri-implant mucosa (Punch Reposition Technique and Topical Flap Technique) are presented and indications outlined. Appreciation of soft tissue conditions as well as functional and esthetic consequences of mucosal deficiencies (mucosal-driven approach) is recommended to supplement bone- and prosthetic-driven considerations in guided oral implant placement (Trinity Approach).

\section{Introduction}

The introduction of computed tomography (CT), virtual implant treatment planning software and $\mathrm{CAD} / \mathrm{CAM}$ technology have undoubtedly been major achievements to provide optimal three-dimensional implant positioning with respect to both prosthetic as well as anatomical parameters. ${ }^{1}$ Mucosa-supported surgical templates offer the possibility of minimally invasive flapless implant surgery associated with significant reduction of intraoperative discomfort and postoperative patient morbidity, ${ }^{2}$ operative time efficiency as well as reduction of surgical error. ${ }^{3}$ Virtual treatment planning may also reduce the need for bone augmentation procedures prior to oral implant placement in the rehabilitation of severely atrophic jaws. ${ }^{4}$ Despite high implant survival rates of $91 \%$ to $100 \%$ reported in clinical studies, ${ }^{5}$ several concerns regarding guided template-based implant dentistry have been raised. Transfer of virtual planning to surgical reality shows inaccuracies of up to $2 \mathrm{~mm}$ and mean angular deviations of $4^{\circ}{ }^{6}$ Heat generation during implant site preparation has been shown to be significantly higher when using surgical templates, ${ }^{7}$ and may lead to osseointegration failure due to thermal bone damage.

Furthermore, the impact of flapless implant placement on peri-implant soft tissue health has been questioned. Significant loss of keratinized mucosa around flapless implants (due to soft tissue punching and removal) can be observed compared to conventional implant placement following mucoperiosteal flaps. ${ }^{8}$ Preclinical research indicates better treatment outcomes if less soft tissue is removed. ${ }^{9}$ Although there is some controversy about if and how much keratinized peri-implant mucosa is required to maintain healthy conditions, shortage or lack of keratinization at least seems disadvantageous in the presence of peri-implant infection. ${ }^{10}$ Guided implant placement using mucosa-supported templates is generally performed in a flapless approach involving soft tissue punching and removal. However, if the amount of available keratinized tissue is limited prior to implant surgery, modified techniques are needed to avoid tissue loss.

\section{Clinical diagnosis of mucosal limits}

Available width of keratinized mucosa may vary significantly depending on jaw location and degree of ridge resorption. In order to identify potential shortcomings at an early stage, it may prove advantageous to visualize intended soft tissue emergence of guided implants in relation to the mucogingival junction. Emergence contour can be marked out by guided soft tissue punching with gentle force (to create only a very shallow cut) after securing the surgical template in its reproducable position (Figure 1A). To investigate mucosal conditions the template may be removed (Figure 1B). If sufficient keratinized mucosa is available around the prospective implant site, the soft tissue can be excised (Figure 1C). In cases of peri-implant mucosal deficiencies, however, deviation from the routine surgical protocol may be considered. Two modified techniques are presented:

\section{Punch reposition technique}

Prior to reinsertion of the surgical template the circular punch incision is extended down to the bone and the soft tissue is carefully elevated and detached (Figure 2A). The punched mucosa (Figure 1D) may be stored in saline solution to prevent dehydration. Surgical steps of implant bed preparation and guided implant
Correspondence: Bernhard Pommer,

Department of Oral Surgery, Vienna Medical University, Sensengasse 2a, A-1090 Vienna, Austria.

Tel: +43.1.40070.4101 - Fax: +43.1.40070.4109

E-mail: bernhard.pommer@meduniwien.ac.at

Key words: minimally invasive surgery, surgical template, guided implant placement, attached mucosa, flapless surgery.

Conflicts of interests: the authors declare no potential conflicts of interests.

Received for publication: 30 September 2011. Revision received: 30 December 2011. Accepted for publication: 23 January 2012.

This work is licensed under a Creative Commons Attribution NonCommercial 3.0 License (CC BYNC 3.0).

(C) Copyright B. Pommer., 2012

Licensee PAGEPress, Italy

Surgical Techniques Development 2012; 2:e7 doi:10.4081/std.2012.e7

placement are then performed according to the routine protocol (Figure 2B). Instead of transmucosal healing, however, a cover screw is placed onto the implant to allow repositioning the punched mucosa to its original location (Figure 1E) secured by 4 to 6 sutures (Figure 2C). Soft tissue healing is generally uneventful and completed within weeks (Figure 1F). The inherent need for second stage surgery after submucosal implant healing may be regarded as the major disadvantage of the Punch Reposition Technique in patient cases that would have qualified for immediate prosthetic restoration otherwise.

\section{Topical flap technique}

Following a curved crestal incision (outline corresponding to the planned implant diameter) a trapezoid buccal flap is raised (Figure 2D) prior to reinsertion of the surgical template. The crestal incision should be shifted to the lingual (palatal) side to include at least 1 $2 \mathrm{~mm}$ of keratinized mucosa in the elevated flap. Surgical steps of implant bed preparation and guided implant placement are then performed according to the routine protocol (Figure 2E) with the buccal flap retracted by the flange of the surgical template. Modification of the inner surface of the template may at times be necessary. The topical flap should be limited to the area of implant emergence, while the divergent releasing incisions allow for sufficient mesiodistal flap width at the base to facilitate perfusion. In 


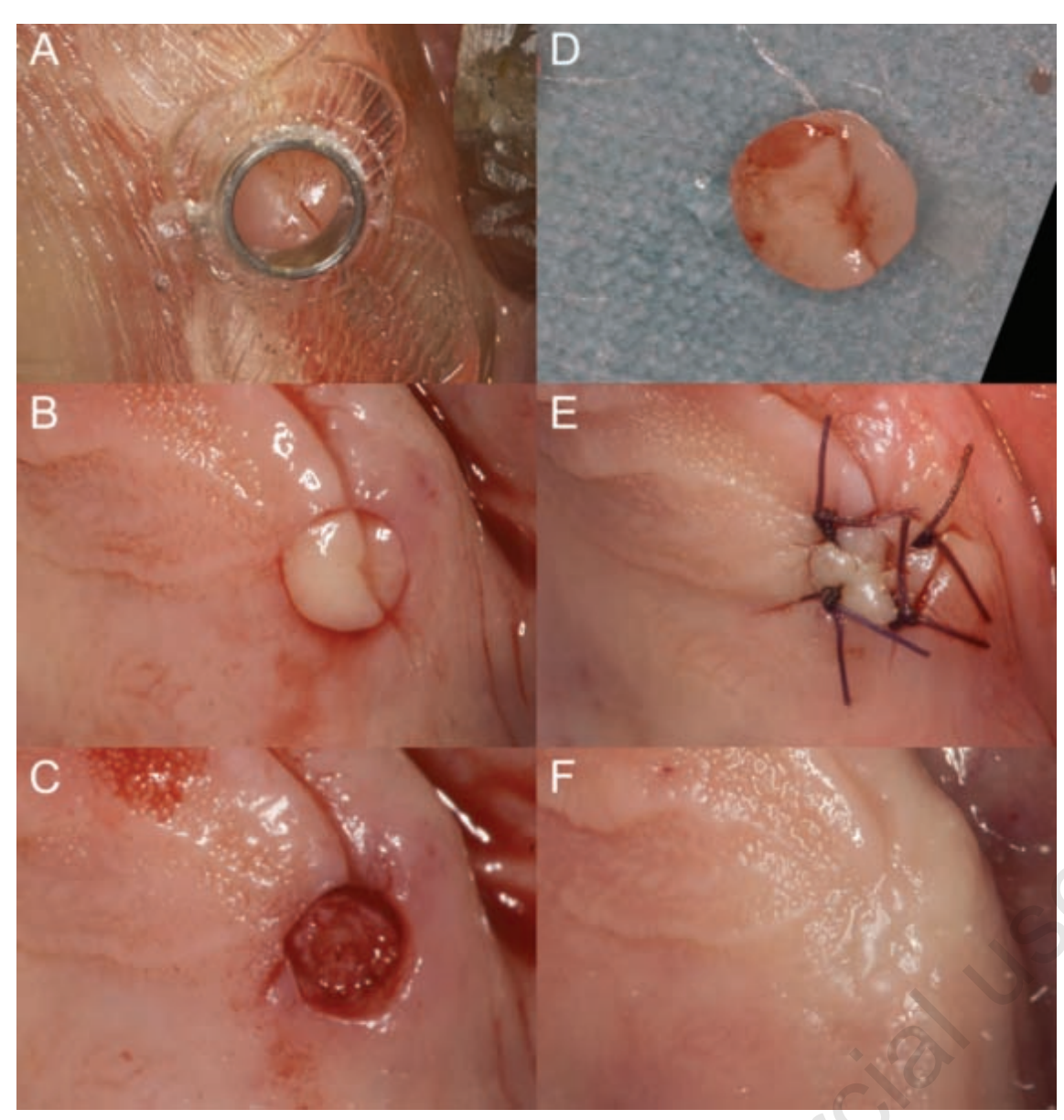

Figure 1. Surgical template (A), guided soft tissue punching (B), soft tissue removal (C), storage of punched mucosa (D), repositioning and suturing $(\mathrm{E})$, soft tissue healing $(\mathrm{F})$.
A

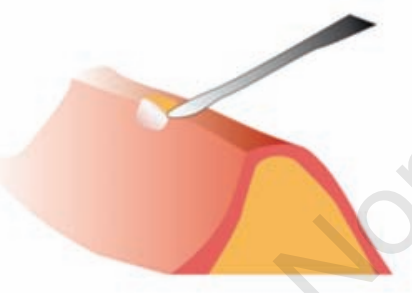

B

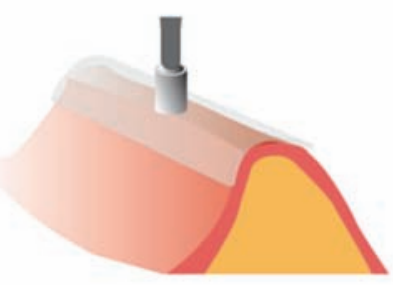

C

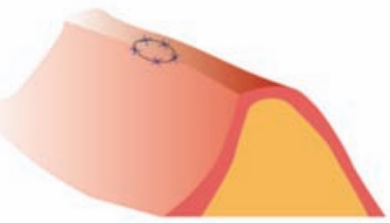

D

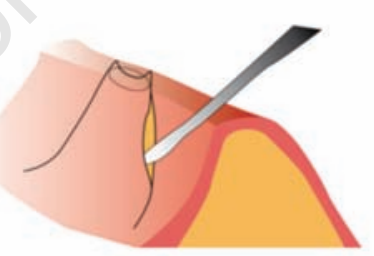

E

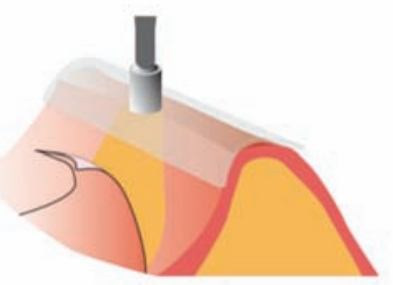

F

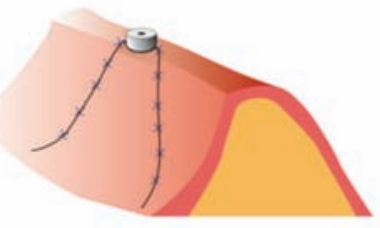

Figure 2. Punch Reposition Technique (A-C), Topical Flap Technique (D-F). cases of mucosal deficiencies at adjacent implants, one combined flap may be raised. The Topical Flap Technique allows for onestage implant surgery using healing abutments and buccal flap repositioning (Figure $2 \mathrm{~F})$. Moreover visual inspection of the buccal cortex during implant site preparation may help to detect peri-implant bone dehiscences. Compared to the Punch Reposition Technique, inherent downsides involve increased surgical invasion and risk of marginal bone resorption due to flap retraction, as well as potential inaccuracy of the mucosa-supported templates (naturally more problematic in complete compared to partial edentulism and mandibular compared to maxillary implants).

\section{Computed tomography-guided treatment planning}

While the two techniques presented may help to preserve keratinized mucosa in cases of limited availability at intended implant sites, additional soft tissue grafts may be inevitable if mucosal deficiencies are severe. However, it seems essential to regard mucosal conditions in CT-guided implant placement (even in the phase of virtual treatment planning) requiring radiographic methods to visualize keratinized mucosa in CT. Appreciation of peri-implant soft tissues in guided implant surgery may be defined as mucosal-driven approach. While bone-demanded implant placement aims at obviating bone graft surgery, ${ }^{11-12}$ and prosthetic-driven implant positioning attempts to reduce the need for compensatory suprastructure design, ${ }^{13}$ mucosaldriven treatment planning is concerned with functional as well as esthetic consequences of peri-implant mucosal deficiencies and avoidance of soft tissue grafts. The conceptual model of co-consideration of bone-, prosthetic-

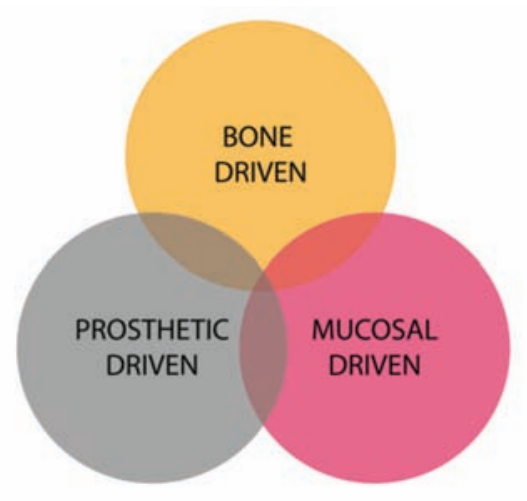

Figure 3. The Trinity Approach: co-consideration of bone-, prosthetic- and mucosaldriven implant placement in guided oral implant surgery. 
and mucosal-driven implant placement in guided oral implant surgery is illustrated in Figure 3.

The Trinity Approach suggests reconciliation of bone-, prosthetic- and mucosal-driven implant placement in CT-guided treatment planning. Prosthetic-demanded implant positions may not always be in agreement with mucosal conditions. However, it needs to be clarified if soft tissue surgery (following prosthetic-driven positioning) or compensatory suprastructure designs (following mucosaldriven placement) should be favored in such cases. In other situations, bone augmentation may prove unavoidable to accomplish implant positions that satisfy mucosal as well as prosthetic needs. As most bone augmentation procedures cannot be performed simultaneously with flapless guided implant placement (with the exception of transcrestal sinus floor elevation $^{14}$ ) CT-based treatment planning must be repeated after bone graft healing. If bone- and prosthetic-demanded implant positions are in compliance and mucosal deficiencies are not severe, the presented techniques to preserve keratinized peri-implant mucosa (Punch Reposition Technique, Topical Flap Technique) may represent the least invasive and most time-efficient treatment option. Comparative clinical research is needed to investigate success and complication rates associated with diverging therapeutic strategies in order to establish a systematic approach to treatment planning for CT-guided oral implant surgery.

\section{References}

1. Jung RE, Schneider D, Ganeles J, et al. Computer technology applications in surgical implant dentistry: a systematic review. Int J Oral Maxillofac Implants 2009;24 Suppl:92-109.

2. Nkenke E, Eitner S, Radespiel-Tröger M, et al. Patient-centred outcomes comparing transmucosal implant placement with an open approach in the maxilla: a prospective, non-randomized pilot study. Clin Oral Implants Res 2007;18:197-203.

3. de Almeida E0, Pellizzer EP, Goiatto MC, et al. Computer-guided surgery in implantology: review of basic concepts. J Craniofac Surg 2010;21:1917-21.

4. Scotti R, Pellegrino G, Marchetti C, et al. Diagnostic value of NobelGuide to minimize the need for reconstructive surgery of jaws before implant placement: a review. Quintessence Int 2010;41:809-14.

5. Schneider D, Marquardt P, Zwahlen M, Jung RE. A systematic review on the accuracy and the clinical outcome of computerguided template-based implant dentistry. Clin Oral Implants Res 2009;20 Suppl 4:7386.

6. Vasak C, Watzak G, Gahleitner A, et al. Computed tomography-based evaluation of template (NobelGuide ${ }^{\mathrm{TM}}$ )-guided implant positions: a prospective radiological study. Clin Oral Implants Res 2011;22:1157-63.

7. Misir AF, Sumer M, Yenisey M, Ergioglu E. Effect of surgical drill guide on heat gener- ated from implant drilling. J Oral Maxillofac Surg 2009;67:2663-8.

8. Van de Velde T, Sennerby L, De Bruyn H. The clinical and radiographic outcome of implants placed in the posterior maxilla with a guided flapless approach and immediately restored with a provisional rehabilitation: a randomized clinical trial. Clin Oral Implants Res 2010;21:1223-33.

9. Lee DH, Choi BH, Jeong SM, et al. Effects of soft tissue punch size on the healing of peri-implant tissue in flapless implant surgery. Oral Surg Oral Med Oral Pathol Oral Radiol Endod 2010;109:525-30.

10. Warrer K, Buser D, Lang NP, Karring T. Plaque-induced peri-implantitis in the presence or absence of keratinized mucosa. An experimental study in monkeys. Clin Oral Implants Res 1995;6:131-8.

11. el Askary A el-S. Esthetic considerations in anterior single-tooth replacement. Implant Dent 1999;8:61-7.

12. Zechner W, Bernhart T, Zauza K, et al. Multidimensional osteodistraction for correction of implant malposition in edentulous segments. Clin Oral Implants Res 2001;12:531-8.

13. Garber DA. The esthetic dental implant: letting restoration be the guide. J Am Dent Assoc 1995;126:319-25.

14. Pommer B, Watzek G. Gel-pressure technique for flapless transcrestal maxillary sinus floor elevation: a preliminary cadaveric study of a new surgical technique. Int J Oral Maxillofac Implants 2009;24:817-22. 